\title{
Broad Histogram Monte Carlo
}

\author{
P.M.C. de Oliveira ${ }^{a}$, T.J.P. Penna ${ }^{a}$ and H.J. Herrmann ${ }^{b}$ \\ a) Instituto de Física, Universidade Federal Fluminense av. Litorânea s/n, Boa Viagem, \\ Niterói RJ, Brazil 24210-340 \\ b) ICA 1, Universität Stuttgart, Pfaffenwaldring 27, 70569 Stuttgart, Germany
}

\begin{abstract}
We propose a new Monte Carlo technique in which the degeneracy of energy states is obtained with a Markovian process analogous to that of Metropolis used currently in canonical simulations. The obtained histograms are much broader than those of the canonical histogram technique studied by Ferrenberg and Swendsen. Thus we can reliably reconstruct thermodynamic functions over a much larger temperature scale also away from the critical point. We show for the two-dimensional Ising model how our new method reproduces exact results more accurately and using less computer time than the conventional histogram method. We also show data in three dimensions for the Ising ferromagnet and the Edwards Anderson spin glass.
\end{abstract}

In the simulation of thermal systems one typically needs to calculate a variable $<Q>_{T}$ as a function of temperature $T$. Usually one would have to perform independent Monte Carlo simulations at different values of temperature. An appealing strategy to avoid such multiple simulations is the canonical histogram method [1,2]: The histogram $P_{T}(E)$ of the energies at one given temperature $T_{0}$ is measured and then the distribution at a different temperature $T$ is obtained by reweighting, i.e. by multiplying with $\exp \left(E / T-E / T_{0}\right)$ and normalizing. In order to obtain the average $\left\langle Q>_{T}\right.$, one needs to accumulate also the histogram of $Q$ as a function of energy $E$. The thermal average at temperature $T$ is then

$$
<Q>_{T}=\sum_{E}<Q(E)>\frac{P_{T_{0}}(E) e^{E\left(T_{0}-T\right) / T_{0} T}}{\sum_{E^{\prime}} P_{T_{0}}\left(E^{\prime}\right) e^{E^{\prime}\left(T_{0}-T\right) / T_{0} T}}
$$

where $<Q(E)>$ means the average value of $Q$ obtained at fixed energy $E$. For simplicity we set $k_{B}=1$ in this article.

The histogram method has been carefully checked on various models [3]. Its main disadvantage is that the canonical distribution $P_{T}(E)$ of the energy is rather narrowly peaked around the average value $\left\langle E>_{T_{0}}\right.$ (and the more so the larger the system) so that when $T$ is not close to $T_{0}$ the reweighting factor is very small there and very large near $\langle E\rangle_{T}$. One also has strong statistical fluctuations stemming from the tails of the distributions. So, in order to get reliable results the tails must be sampled very well by making good statistics and the temperatures considered should not be far from $T_{0}$.

We want to introduce here a completely different technique based on the calculation of the degeneracy $g(E)$ of energy states from histograms of adequate macroscopic quantities defined below [4]. Figure 1 compares the classical canonical histogram for an Ising model on 
square lattices to the one obtained with our method. As mentioned before the width of the classical histogram method decreases with system size. Instead the histogram introduced in the following covers the entire energy range.

The first step of our method [4] is to perform a Markovian walk along the energy axis which samples all energies with the same weight. For that we define two classes of moves in phase space:

$$
\begin{array}{ll}
\text { class } 1: & E \longrightarrow E-\Delta E \\
\text { class 2: } & E \longrightarrow E+\Delta E,
\end{array}
$$

where $\Delta E>0$. To correctly sample $g(E)$ we propose the following dynamics: We randomly choose a move. If this move belongs to class 1 , it is accepted. If, however, it belongs to class 2 , it is accepted only with probability $N_{\text {dn }} / N_{\text {up }}$, where $N_{\text {dn }}$ and $N_{\text {up }}$ are the total numbers of possible moves of classes 1 and 2, respectively, measured at the current state.

These quantities $N_{\mathrm{dn}}$ and $N_{\text {up }}$ concern the whole lattice, considering all possible movements one can perform at the current state in order to get a (would-be) next state along the Markovian chain. In the particular case of a single spin flip dynamics, $N_{\text {dn }}$ and $N_{\text {up }}$ are obtained by checking on each site if a spin flip would increase or decrease the energy and counting how many times each case occured. Then one site is chosen randomly and the above rule is applied only to it. Nevertheless, the method is not restricted to single spin flips, and any other updating protocol can be adopted, provided all possible movements are classified as belonging to classes 1 or 2 , in order to count their current numbers $N_{\mathrm{dn}}$ and $N_{\text {up }}$, before actually performing one of them. Following this rule the probabilities to increase or to decrease the energy are equal.

Like for a random walk, the region already visited along the energy axis increases proportionally to $\Delta E \sqrt{t}$, where $t$ is the number of performed moves, i.e. the length of the Markovian sequence of states. This allows one to obtain any predefined distribution width, simply by taking a large enough computer time. On the other hand, in canonical dynamics based on the Boltzmann weights one always get distributions with exponential decaying tails, and thus with time-independent widths. Figure 1 shows the number of visits as a function of the energy.

The second step of our method is the calculation of the degeneracy of energy states $g(E)$. In the above Markovian process, the probability for the energy to jump from $E$ to $E+\Delta E$ is the same as that of jumping back from $E+\Delta E$ to $E$ :

$$
<N_{\text {up }}(E)>g(E)=<N_{\mathrm{dn}}(E+\Delta E)>g(E+\Delta E)
$$

where the averages are taken at fixed energy. Equation (2) can be rewritten as

$$
\beta(E) \equiv \frac{\mathrm{d} \ln g(E)}{\mathrm{d} E}=\frac{1}{\Delta E} \ln \frac{<N_{\mathrm{up}}(E)>}{<N_{\mathrm{dn}}(E+\Delta E)>},
$$

In this way one obtains $g(E)$, from the averages $\left\langle N_{\text {up }}(E)>\right.$ and $\left\langle N_{\mathrm{dn}}(E)>\right.$ accumulated during the random walk.

Summarizing, the method consists in performing the Markovian process defined above, and accumulating four histograms along the $E$ axis: for the number of visits; for the 
quantity $Q$ one is interested in; for the average number $N_{\mathrm{dn}}$ of moves of class 1 ; and for $N_{\text {up }}$ corresponding to class 2. From these numbers, $g(E)$ is determined by equation (3), and the thermal average $\langle Q\rangle_{T}$ obtained through equation (1) using

$$
P_{T}(E)=\frac{1}{Z_{T}} g(E) \exp (-E / T)
$$

with $Z_{T}=\sum_{E} g(E) \exp (-E / T)$, if one wants to work in the canonical ensemble.

For the one-dimensional Ising model we have been able to prove that our model gives the exact solution for $g(E)$ [4]. Also in this same reference one can find a generalization of equation (3) for the case where different values of $\Delta E$ are possible: it is enough to transfer the factor $1 / \Delta E$ as an exponent in both the numerator and denominator, inside the average brackets.

In order to test our new technique numerically we performed simulations for three examples: the two- and three-dimensional Ising ferromagnet and the 3D Ising spin glass. Starting with a random configuration we choose in the ferromagnetic cases an initial state that was thermalized at the critical temperature for 10 Metropolis lattice sweeps [5]. For the spin glass we did instead 100 sweeps at zero temperature. We checked, however, that the results did not depend on the initial state. Our systems had periodic boundary conditions in all directions. For efficiency we used the multilattice approach [6] storing 32 lattices in an array of 32-bit integers. All 32 samples are processed in parallel by using multi-spin coding [7].

The degeneracy $g(E)$ of states obtained for the two-dimensional Ising model on a $32 \times 32$ square lattice agrees so well with the recently derived exact degeneracy [8] that on a plot they are indistinguishable from each other. Figure 2 shows the averaged energy and specific heat, obtained by the present method. The data are extremely close to the exact curves [9]. This is true even for the specific heat at its maximum where statistical fluctuations are largest as can be seen from the inset of figure 2 .

In figure 2 we also see data obtained using the classical canonical histogram method $[1,2]$. The data noticeably deviate around the maximum of the specific heat which is probably due to the fact that the width of the canonical histogram is not larger than the distance between this maximum and $T_{c}$, the temperature at which the histogram was calculated. Although the computational effort invested in CPU time was about $50 \%$ larger for our method than for the canonical histogram method (using in both cases the same multi-spin implementation) the result obtained with our method is at least ten times more precise. Moreover, for the canonical histogram technique one must simulate independently at several temperatures to get the data in the temperature range considered. This is a convincing evidence for the efficiency of our method.

For the two-dimensional Ising ferromagnet the magnetization [10] and susceptibility were also analyzed and compared with canonical Monte Carlo simulations [11]. The agreement is again excellent. We did the same studies also for the three-dimensional Ising model. Again energy, specific heat, magnetization and susceptibility were calculated and found in very good agreement with known results investing a quite modest computational 
effort. In figure 3 we show as an example magnetization and susceptibility for 3D systems differing in size. For large lattices like the $50 \times 50 \times 50$ presented in figure 3 , the random walk dynamics may be very slow, if one starts always from only one energy. In these cases, it is better to restart the dynamics many times during the simulation, each time choosing a new value for the starting energy. This trick was adopted also for the $256 \times 256$ lattice in figure 1.

As a more difficult test we chose also to simulate the three-dimensional Ising spin glass. Besides energy and specific heat we also calculated the overlap order parameter defined through

$$
q=\left[\frac{1}{N} \sum_{i}<s_{i} s_{i}^{(0)}>_{T}\right]_{a v}
$$

where $\left\{s_{i}^{(0)}\right\}$ is a ground state previously determined by the approximation introduced in [12]. In figure 4 we show that it goes to zero close to the predicted temperature [13]. Again the method turns out to be very efficient.

Two last points deserve comments. First, there are other methods [14] which can be thought as similar to our first step, where we defined a non-biased random walk dynamics. All of them introduce non-canonical ensembles directly weighted by the unknown entropy, or $g(E)$. Karliner et al [14], for instance, use the $g(E)$ scaled up from exact calculations on small lattices in order to define the statistical weight of their dynamics, replacing the Boltzmann factors. In all these earlier works, the entropy or $g(E)$ is measured directly from the accumulated number of visits to each energy channel, within the particular dynamics corresponding to each case. Our approach is distinct from those others: we do not use the number of visits, on the contrary, our results are extracted from accumulated histograms of quantities measured within each visited state, namely $N_{\mathrm{dn}}$ and $N_{\text {up }}$, concerning the whole lattice. The only role played by the number of visits to each energy channel is that it must be large enough to provide a good statistics. Being based on statistical averages of macroscopic quantities, our method also has a better numerical precision. Indeed, accumulating the macroscopic quantities $N_{\mathrm{dn}}$ and $N_{\mathrm{up}}$ (both of the order of the number of sites), instead of merely incrementing by unity a visit counter after each movement as in the traditional histogram method, we succeed in getting better numerical accuracy.

Second, the left hand side of equation (3) coincides with the formal definition of (inverse) temperature as a function of the energy $E$. Thus, the averages on the right hand side of the same equation correspond to the canonical ensemble equilibrium. Although in our second step we have derived equation (3) from the particular dynamics defined in the first step (the non-biased random walk), it holds for any other dynamics respecting the canonical equilibrium for each (inverse) temperature $\beta(E)$. In this sense, the importance of our method relies on the second step, the determination of $g(E)$ from the countings of potential movements $N_{\mathrm{dn}}$ and $N_{\mathrm{up}}$, measured within any canonical equilibrium dynamics.

We have presented a new histogram Monte Carlo method which as compared to the traditional one based on temperature $[1,2]$ is based on the histograms of the energy. These 
histograms have the advantage of having much broader tails allowing to extrapolate to a much larger range of temperatures with a rather small number of samples. We have tested our method on the two- and three-dimensional Ising model and the 3D Ising spin glass and succeeded in reproducing thermodynamic quantities with higher accuracy over a larger temperature scale and with less computational effort than the canonical histogram method.

We thank L. de Arcangelis, D. Stauffer, S. Moss de Oliveira, C. Moukarzel and YiCheng Zhang for helpful discussions.

\section{References}

[1] Salzburg Z.W., Jacobson J.D., Fickett W. and Wood W.W., J. Chem. Phys. 3065 (1959).

[2] Ferrenberg A.M. and Swendsen R.H., Phys. Rev. Lett. 61, 2635 (1988).

[3] Ferrenberg A.M. and Landau D.P., Phys. Rev. B44, 5081 (1991).

[4] P.M.C. de Oliveira, T.J.P. Penna and H.J. Herrmann, Braz. J. of Physics 26, 677 (1996).

[5] In some cases it was also useful to perform one or several Metropolis updates after each iteration of the Markov process at the temperature given by equation (3) in order to reduce correlations and accelerate the algorithm.

[6] Bhanot G., Duke D. and Salvador R., J. Stat. Phys. 44, 985 (1986); Phys. Rev. B33, 7841 (1986).

[7] de Oliveira P.M.C., Computing Boolean Statistical Models World Scientific, Singapore, ISBN 981-02-0238-5 (1991).

[8] Beale P.D., Phys. Rev. Lett. 76, 78 (1996).

[9] Ferdinand A.E. and Fisher M.E., Phys. Rev. 185, 832 (1969).

[10] In order to break the global spin flip symmetry, the magnetization was in fact defined as the average of the absolute difference between the population fractions of spins up and down.

[11] see, for instance, Landau L.D., Phys. Rev. B13, 2997 (1976), or de Oliveira P.M.C. and Penna T.J.P., Rev. Bras. Fís. 18, 502 (1988).

[12] Stauffer D. and de Oliveira P.M.C. Physica A215, 407 (1995).

[13] A.T. Ogielski, Phys. Rev. B 32, 7384 (1985), N. Kawashima and A.P. Young, Phys. Rev. B 53, R484 (1996).

[14] B. Bhanot, S. Black, P. Carter and S. Salvador, Phys. Lett. B183, 381 (1987); M. Karliner, S. Sharpe and Y. Chang, Nucl. Phys. B302, 204 (1988); B. Berg and T. Neuhaus, Phys. Lett. B267, 249 (1991); Phys. Rev. Lett. 68, 9 (1992); B. Berg, Int. J. Mod. Phys. C3, 1083 (1992); B. Berg, T. Celik and U. Hansmann, Europhys. Lett. 22, 63 (1993); J. Lee, Phys. Rev. Lett. 71, 211 (1993); B. Hesselbo and R.B. Stinchcombe, Phys. Rev. Lett. 74, 2151 (1995). 


\section{Figure Captions}

Figure 1 Number of visits as a function of energy obtained from the traditional histogram method [1,2] (fixing the temperature at the critical value), and from the present method, for the Ising ferromagnet on $32 \times 32$ and $256 \times 256$ square lattices. The energy is scaled such that the critical value corresponds to 0.146. Energies above $\sim 0.4$ are not sampled for the $256 \times 256$ lattice, because they do not contribute to thermal averages at the interesting range of temperatures $\left(0<T<2 T_{c}\right)$.

Figure 2 Averaged energy and specific heat obtained from the present method for the Ising ferromagnet on a $32 \times 32$ square lattice and the exact ones [8] (both full lines). Except for a small region around the critical point (see inset for maximum of specific heat) the two curves are indistinguishable. Results of similar quality were also obtained for larger lattices (we tested $L=64,128$ and 256). The open circles represent data obtained using the canonical histogram method $[1,2]$ for which $P_{T}(E)$ was obtained at $T_{c}$. Our method needed just 40 minutes on a Pentium $(66 \mathrm{MHz})$ for the $6 \times 10^{4}$ lattice sweeps performed.

Figure 3 Magnetic susceptibility obtained from the present method for the 3D Ising ferromagnet on a $50 \times 50 \times 50$ (upper curves, showing also the magnetization), a $20 \times 20 \times 20$ (middle curve) and a $10 \times 10 \times 10$ (lower curve) cubic lattice.

Figure 4 Overlap with one specific previously calculated low temperature state and non-linear susceptibility of this order parameter for the 3D Ising spin glass simulated on a $8 \times 8 \times 8$ cubic lattice making 53000 lattice sweeps for 128 different configurations of couplings. The total amount of CPU time spent to get these data was 15 hrs on a Pentium $(66 \mathrm{MHz})$. 


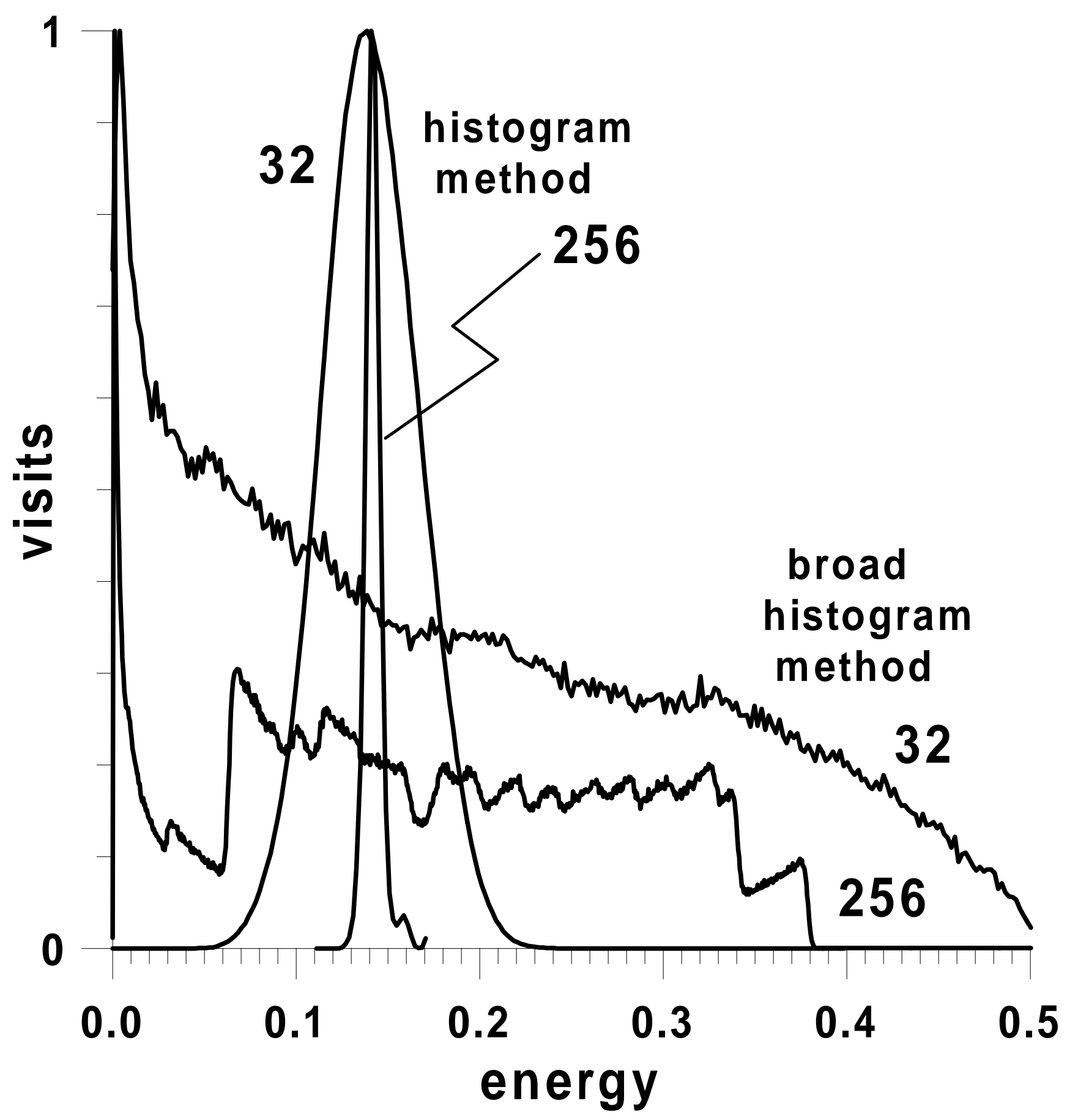




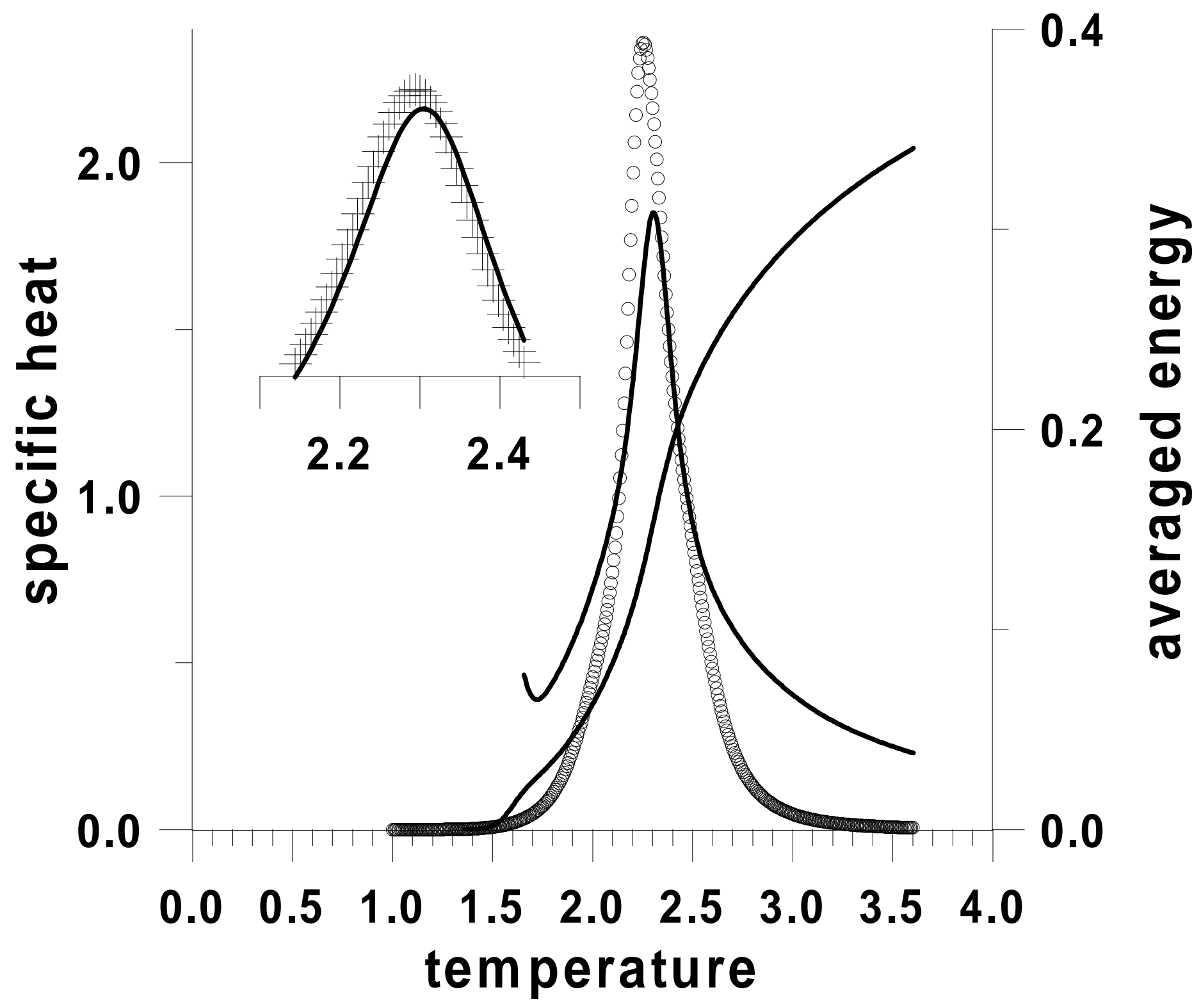




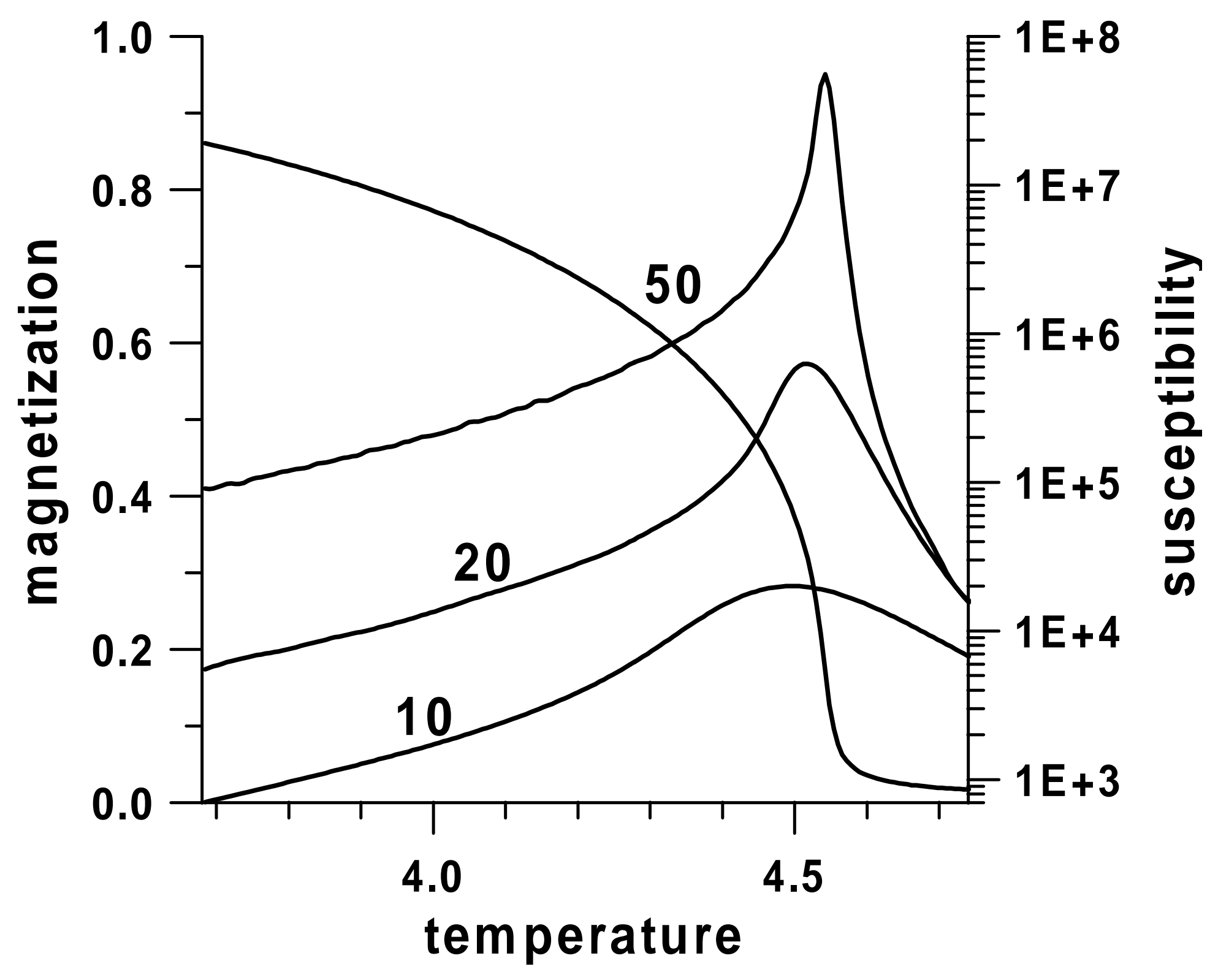




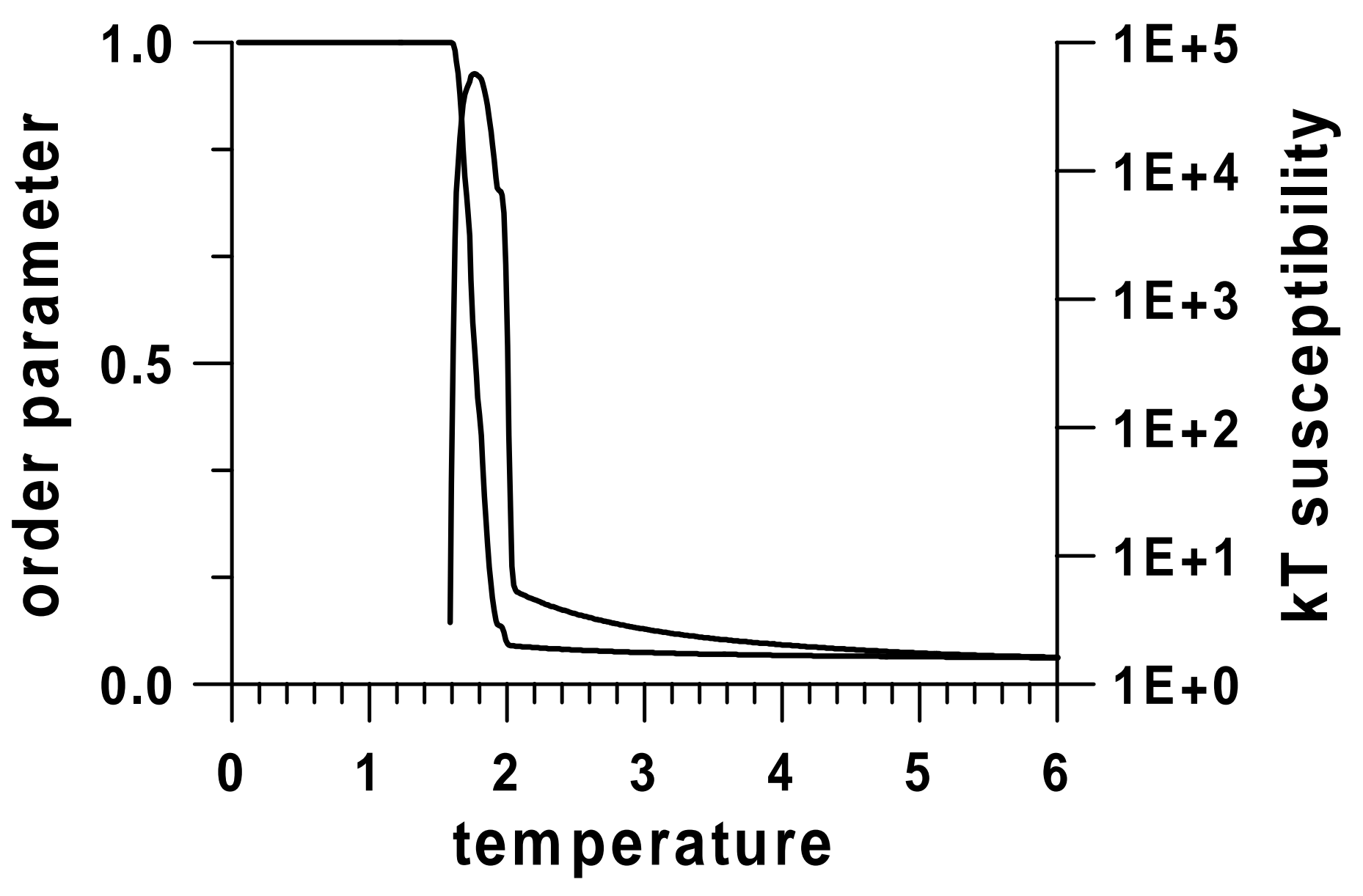

